\title{
COMMUNICATION
}

\section{Nodule vestibulaire maxillaire : à propos d'un cas atypique de tumeur odontogène}

\author{
Pajot $\mathrm{T}^{1,2}$, Genevière $\mathrm{H}^{3,4}$, Descroix $\mathrm{V}^{5}$, Lescaille $\mathbf{G}^{5,6}$ \\ 1. Service d'Odontologie, Groupe Hospitalier Pitié-Salpêtrière, Assistance Publique-Hôpitaux de Paris (AP-HP) - Paris \\ 2. Université Paris XII (UPEC) - Université Paris-Est Marne-la-Vallée (UPEMLV) \\ 3. Sorbonne Universités UPMC - Paris 06 \\ 4. Service d'Anatomopathologie, Groupe Hospitalier Pitié-Salpêtrière, Assistance Publique-Hôpitaux de Paris (AP-HP) - Paris \\ 5. Université Paris Diderot - Sorbonne Paris Cite - Paris 07 \\ 6. Centre d'Immunologie et Maladies Infectieuses (CIMI-Paris), UMR-S INSERM U1135, CNRS ERL 8255, \\ Sorbonne Universités, UPMC - Paris 06
}

\section{Introduction}

Les tumeurs odontogènes appartiennent à la catégorie des tumeurs osseuses des maxillaires. Elles sont de différentes natures selon leur origine épithéliale, mésenchymateuses ou mixte, très majoritairement bénignes, rarement cancéreuses. Ces lésions sont le plus souvent découvertes de manière fortuite à l'imagerie, mais peuvent dans certains cas prendre une localisation périphérique gingivale. Le cas d'une patiente présentant un nodule gingival correspondant à une tumeur odontogène est rapporté ici.

\section{Observation}

II s'agissait d'une patiente âgée de 21 ans adressée par son ORL, pour la prise en charge d'un nodule muqueux d'évolution lentement progressive. Cette patiente présentait depuis plusieurs années une tuméfaction vestibulaire dans le secteur 1 devenu gênante du fait de son augmentation de taille ces derniers mois. À l'examen clinique, on notait la présence d'un volumineux nodule sessile vestibulaire d'environ $2 \mathrm{~cm}$ de diamètre en regard de la dent 16 , ferme, ferme et recouvert d'une muqueuse normale, sans anomalie dentaire particulière associée. Les diagnostics différentiels de fibrome, fibrome à cellules géantes, fibrome périphérique odontogène, fibrome desmoïde, lipome ont été principalement évoqués. Le CBCT montrait une image radioclaire bien limitée et multiloculaire s'étendant en vestibulaire de 16 , avec persistance d'une corticale très fine. Compte tenu de l'absence de signes clinique et radiologique de malignité, l'exérèse complète de la lésion sous anesthésie locale a été décidée. Un lambeau vestibulaire de pleine épaisseur par incision intrasulculaire s'étendant de 14 à 17 a été levé en réalisant une dissection de la paroi du nodule en vestibulaire avec la muqueuse gingivale. L'exérèse de la lésion a été réalisée et la pièce envoyée pour une analyse histologique qui a conclu au diagnostic de fibrome odontogène périphérique. Les suites opératoires ont été simples, il persistait une légère récession gingivale en vestibulaire de 16 sans autre anomalie parodontale à 6 mois avec une cicatrisation osseuse complète au CBCT de contrôle.

\section{Discussion}

Les tumeurs odontogènes périphériques sont rares avec peu d'étude de cohorte dans la littérature. II semble toutefois que le fibrome odontogène périphérique (PODF), dont l'origine serait parodontale et qui serait un variant du fibrome odontogène central, soit la plus fréquente de ces tumeurs devant l'améloblastome périphérique et la tumeur odontogène kystique calcifiée. Le PODF se présente sous la forme d'une masse ferme et sessile de croissance lente et est recouverte d'une muqueuse normale dont les localisations divergent.

\section{Conclusion}

\|l s'agit donc ici d'un cas atypique de fibrome odontogène périphérique qui rappelle que devant la présence d'une lésion nodulaire gingivale, un examen radiologique mais également anatomopathologique est nécessaire pour réaliser un diagnostic précis. Bien que les récidives soient rares, le suivi de ces patients est nécessaire, notamment lorsque la dent en regard de la lésion est conservée comme c'est le cas ici.

th.pajot@hotmail.fr

\section{Références}

Buchner A1, Merrell PW, Carpenter WM. Relative frequency of peripheral odontogenic tumors: a study of 45 new cases and comparison with studies from the literature. J Oral Pathol Med. 2006 Aug;35(7):385-91. Kumar R, Jaiswal S, Sharma A, Andhare V, Sabir H. Peripheral odontogenic fibroma: a rare gingival neoplasm with clinico-pathological differential diagnosis. J Exp Ther Oncol. 2015;11(1):17-21. Reddy SV, Medikonda SK, Konda A, Natta S.A rare benign odontogenic neoplasm: peripheral odontogenic fibroma. BMJ Case Rep. 2014 Jun 11;2014.

(C) The authors, published by EDP Sciences. This is an Open Access article distributed under the terms of the Creative Commons Attribution License 4.0 (http://creativecommons.org/licenses/by/4.0/). 